\title{
A study on the micromechanical behavior of Ti-55531 titanium alloy with lamellar microstructure by in-situ neutron diffraction
}

Yimin $\mathrm{Cui}^{\mathrm{a}}$, Chaohua $\mathrm{Li}^{\mathrm{a}}$, Stefanus Harjo ${ }^{\mathrm{b}}$, Changsheng Zhang ${ }^{\mathrm{c}}$, Runguang $\mathrm{Li}^{\mathrm{a}}$, Weiwei Zheng ${ }^{\mathrm{a}}$, Yandong Wang ${ }^{\mathrm{a}}$

${ }^{a}$ Beijing Advanced Innovation Center for Materials Genome Engineering, State Key Laboratory for Advanced Metals and Materials, University of Science and Technology Beijing, Beijing 100083, China

b J-PARC Center, Japan Atomic Energy Agency, 2-4, Shirane Shirakata, Tokai, Ibaraki 315-1195, Japan

${ }^{\mathrm{c}}$ Institute of Nuclear Physics and Chemistry, China Academy of Engineering Physics, Mianyang 621999, China

\section{$\underline{\text { Abstract }}$}

High strength titanium alloys are promising materials for heavy component parts in the aviation industry. The limited combination of strength and ductility requires an understanding of deformation and stress partitioning between constituent phases. The micromechanical behaviors of Ti-55531 titanium alloy with lamellar microstructure are investigated by in-situ neutron diffraction. The phase strain and lattice strain evolution of $\alpha$ and $\beta$ phase at loading direction and transverse direction are determined. The results show that the micromechanical behaviors of oriented grains of $\alpha$ and $\beta$ phase are obviously different. Furthermore, the stress partitioning between $\alpha$ and $\beta$ phase during the deformation is clearly illustrated. It is found that the $\beta$ matrix is subjected to higher stress than $\alpha$ precipitates. In addition, the intergranular and interphase microstress is quantitatively characterized. It is found that the intergranular microstress in the $\beta$ phase grains is predominant among these microstresses. Combining the in-situ neutron diffraction with microstructure characterization, the present work provides a guide for further microstructure optimization.

\section{Introduction}

High strength titanium alloys are the most promising materials to manufacture key component parts in aviation as excellent mechanical properties such as high specific strength and excellent corrosion resistance [1]. A large amount of literatures have focused on the size, shape and distribution of $\alpha$ precipitates on the mechanical properties of titanium alloys. For instance, it is found that the main microstructure characteristic which shows an important effect on the mechanical properties of titanium alloys with lamellar microstructure (LM) is the size of $\alpha$ lamella colony. The titanium alloys with basket-weave microstructure with a small colony size exhibit higher strength and fracture toughness [2]. The grain boundary $\alpha$ layer is recognized as a detrimental factor that could decrease the ductility of LM. Besides $\alpha$ phase precipitates, S. Hemery et al. have evidenced the highly heterogeneous strain field using the in-situ SEM tensile test and EBSD characterization. The elastic anisotropy of $\beta$ phase is identified as the root cause [3]. Neutron diffraction characterization proves to be a powerful tool to probe the micromechanical behaviors of metals and alloys. Our previous work has shown the micromechanical behaviors of duplex phase steels by in-situ neutron and high energy X-ray diffraction [4]. For titanium alloys, many literatures have focused on the relationship between microstructures and mechanical properties [5], however, there still lacks a fundamental understanding of the role that each constituent phase play during the deformation. Therefore, the main purpose of present work is to investigate the micromechanical behaviors of constituent phases and stress partitioning between $\alpha$ and $\beta$ phase by in-situ neutron diffraction. Combining with microstructure characterization, stress and strain partitioning mechanism between $\alpha$ and $\beta$ phase would be discussed in detail.

\section{Material and experiments}

The nominal chemical composition of as-received Ti-55531 alloy is Ti-5Al-5Mo-5V-3Cr-1Zr, mass \%. Cylindrical specimens of $100 \mathrm{~mm}$ in length and $12 \mathrm{~mm}$ in diameter were machined from the as-received materials. In order to obtain $\mathrm{LM}$, the cylindrical rods were subjected to the following heat treatment: solid solution at $860{ }^{\circ} \mathrm{C}$ for 30 minutes, air cooling, and ageing at $600^{\circ} \mathrm{C}$ for $2 \mathrm{~h}$, air cooling. The neutron diffraction profile (see Fig. 1a) shows that LM consists of $\beta$ phase (BCC structure) and $\alpha$ phase (HCP structure). The volume fraction of $\alpha$ and $\beta$ phase could be obtained by the Rietveld method using Z-Rietveld [6]. The volume fraction of $\alpha$ phase is equal to $58 \pm 2 \%$. Consequently, the volume fraction of $\beta$ phase is $42 \pm 2 \%$. Fig. $1 \mathrm{~b}$ shows the micrograph of LM. It is shown that the $\alpha$ lamellas are dispersed in the $\beta$ matrix. The rod tensile specimen for in-situ neutron diffraction experiment with a gauge length of $8 \mathrm{~mm}$ in length, $4 \mathrm{~mm}$ in diameter was machined from cylindrical specimens after heat treatment.
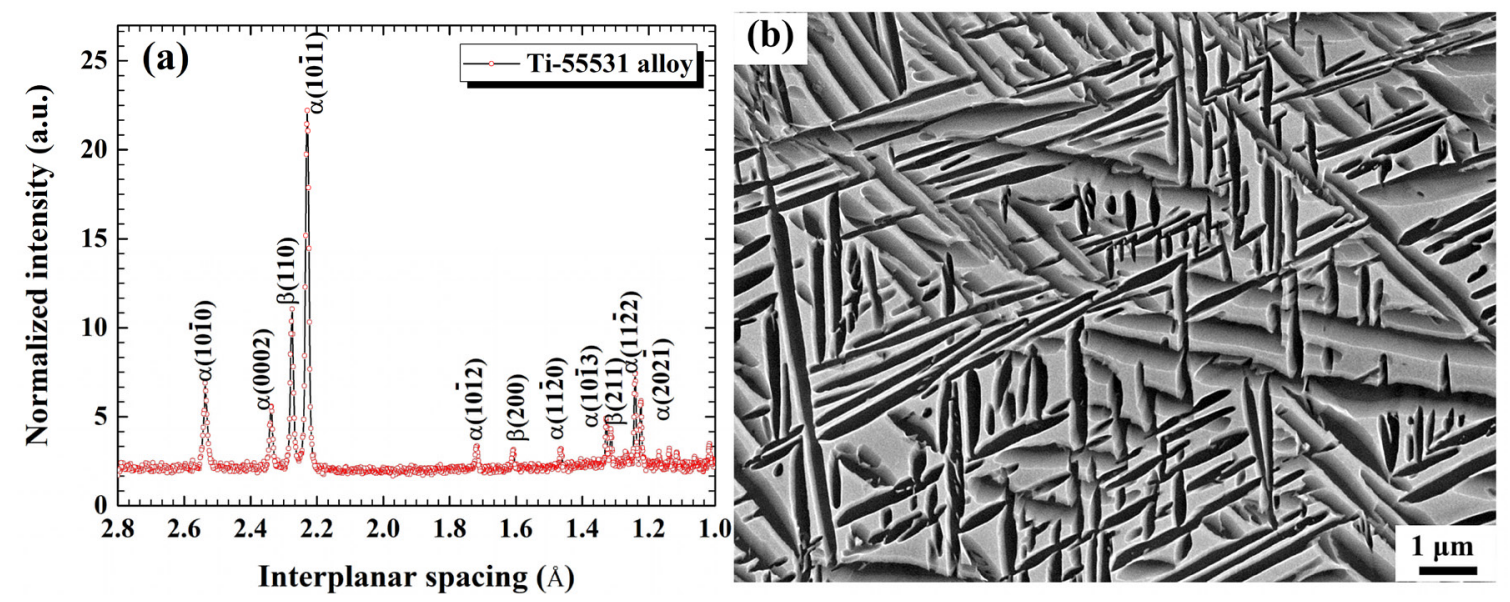

Fig. 1 The initial microstructure of Ti-55531 alloy: (a) neutron diffraction profile prior to loading, and (b) SEM image of LM.

The in-situ neutron diffraction experiment was conducted at TAKUMI, a high resolution and high intensity time of flight (TOF) neutron diffractometer for engineering materials science at Materials and Life Science Experimental Facility of Japan Proton Accelerator Research Complex (J-PARC). The set-up of in-situ neutron diffraction instrument is illustrated in Fig. 2a. The incident neutron beam was restricted to $(5 \times 5) \mathrm{mm}$ by incident silts. North and south detector banks were employed to collect the diffraction patterns at the radial (transverse direction-TD) and axial direction (loading direction-LD) of the specimen, respectively. The neutron diffraction data were collected continuously using an event-recording mode during tensile deformation. The measurement time was 30 min for each measurement. During the experiment, the specimen was subjected to tensile loading at a constant crosshead displacement rate of $0.12 \mathrm{~mm} / \mathrm{s}$ at room temperature. Fig. $2 \mathrm{~b}$ shows the measured macroscopic stress-strain curve of Ti55531 alloy with LM. The notable stress relaxation was found during the plastic deformation. The whole diffraction profiles were fitted using the Rietveld method to obtain lattice parameters, thus the phase strain $\left(\varepsilon_{p h}\right)$ could be expressed as:

$$
e^{p h}=\left(a^{\text {ph }}-a_{0}^{\text {ph }}\right) / a_{0}^{\text {ph }}
$$


where ${ }_{0}^{p h}$ represents the reference lattice parameter $(p h=\alpha, \beta)$. The interplanar spacings of $\{h k l\}$ reflections could be determined by fitting each individual peak using pseudo-voigt function. The lattice strain $\left(\varepsilon_{h k l}\right)$ was thus determined according to the variation of interplanar spacings,

$$
\epsilon_{h k I}=\left(d_{h k I}-d_{h k l}^{0}\right) / d_{h k I}^{0}
$$

where $d_{h k I}^{\circ}$ represents interplanar spacing of considered $\{h k l\}$ reflections prior to tensile loading. The EBSD orientation analysis was carried out to characterize the microstructure and orientation of deformed tensile specimens. The EBSD sample was prepared by electrolytic polishing followed mechanical grinding. EBSD analysis was conducted on a ZEISS SUPRA 55 SEM equipped with Channel 5 system.
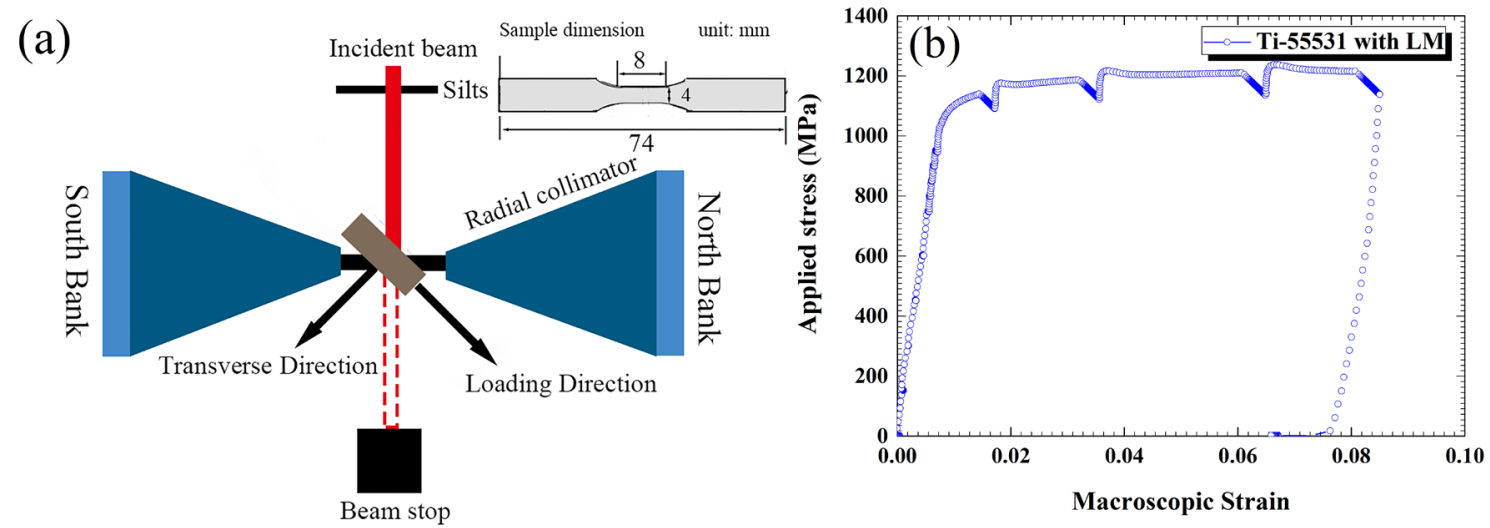

Fig. 2 in-situ neutron diffraction characterization: (a) set-up of in-situ neutron diffraction instrument, and (b) macroscopic stress-strain curve obtained during in-situ loading experiment.

\section{$\underline{\text { 3. Results }}$}

3.1 The lattice strain evolution during the deformation

The lattice strain evolution gives the -dependent micromechanical behavior. Fig. 4 plots the lattice strain, $\varepsilon_{h k l}$, as a function of applied stress. According to the characteristic of $\sigma-\varepsilon_{h k l}$ curves, the deformation process could be divided into two stages. In stage I, the lattice strain of selected oriented grains responds linearly to the applied stress at both LD and TD. The slop of $\sigma-\varepsilon_{h k l}$ curves represents -dependent elastic property. In accordance with other BCC materials, $<200>$ direction shows the lowest stiffness. When the deformation enters into stage II, notably deviation from linearity of lattice strain is observed in all the considered \{ $h \mathrm{kl}\}$ reflections. The lattice strain evolution of oriented grains is obviously different in stage II. The lattice strain of $\langle 200\rangle$ oriented grains keeps increasing during the plastic deformation, while there are only a slight increase observed in the lattice strain of $\langle 110>$ and $<211>$ oriented grains. With regard to the hexagonal $\alpha$ phase, $<0002>$ orientation exhibits the highest stiffness during the elastic deformation. The continuous increase of lattice strain is observed in the $\{0002\}$ and $\{1010\}$ reflections. The evident difference in lattice strain evolution between different oriented grains suggests the stress transfer between different oriented grains which would be discussed in detail later. At the TD, compressive lattice strain is observed as the specimen is subjected to compressive stress in this direction. Particularly, the lattice strain of $\langle 200\rangle$ oriented grains at the TD begins to increase in stage II, which indicates that the applied stress has been transferred to other oriented grains.

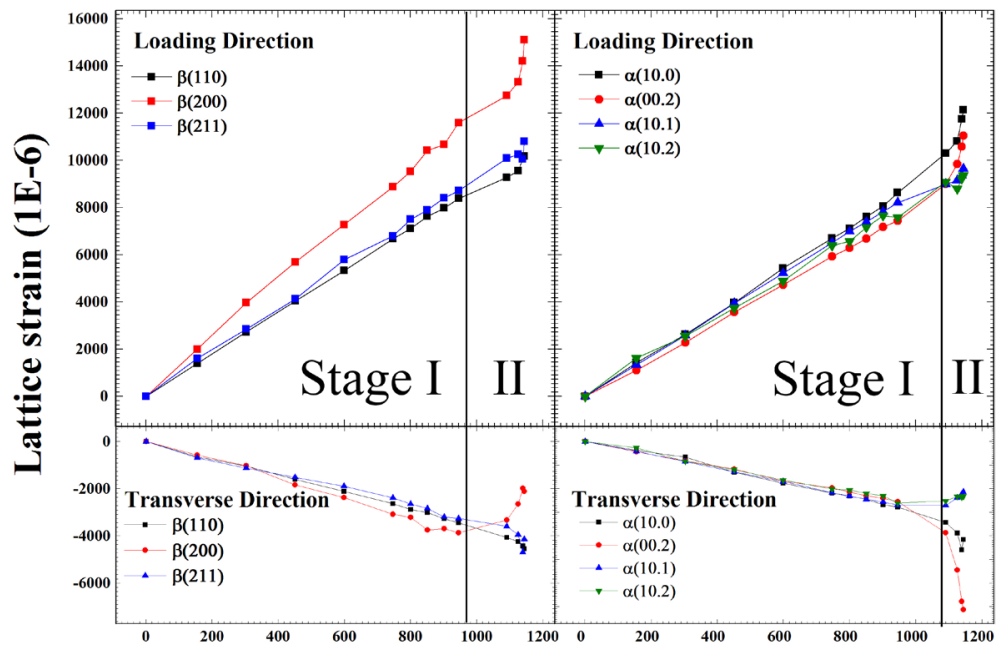

Applied stress (MPa)

Fig. 3 The lattice strain vs. applied stress for considered $\{h k l\}$ reflections of $\beta$ and $\alpha$ phase, in each constituent phase, the lattice strain at the LD and TD is shown.

As the anisotropy deformation behavior of oriented grains is balanced by the Rietveld method, the phase strain evolution could provide valuable information relating to the micromechanical behavior of $\alpha$ and $\beta$ phase as a whole. The phase strain is obtained by fitting the diffraction profiles at the LD and TD using the Riveted method. The results give the lattice parameters at each measurement point. The phase strain is plotted as a function of applied stress in Fig. 3. In stage I, both the phase strain of $\alpha$ and $\beta$ phase responds linearly to the applied stress, which indicates elastic deformation is the main deformation mode. The slop of stress-strain curves represents the elastic property of $\alpha$ and $\beta$ phase, respectively. It is found that the $\alpha$ phase presents higher stiffness than $\beta$ phase during elastic deformation. At stage II, the nonlinear lattice strain appears at both the LD and TD. At the LD, the continuous increase of phase strain of $\beta$ phase is observed, while there is a slight decrease in the phase strain of $\alpha$ phase. At the TD, the magnitude of phase strain of $\alpha$ phase starts to decrease at stage II, while the strain of $\beta$ phase keeps increasing during the plastic deformation. These findings suggest stress partitioning exists between $\alpha$ and $\beta$ phase which is important to the mechanical properties of Ti-55531 alloy. 


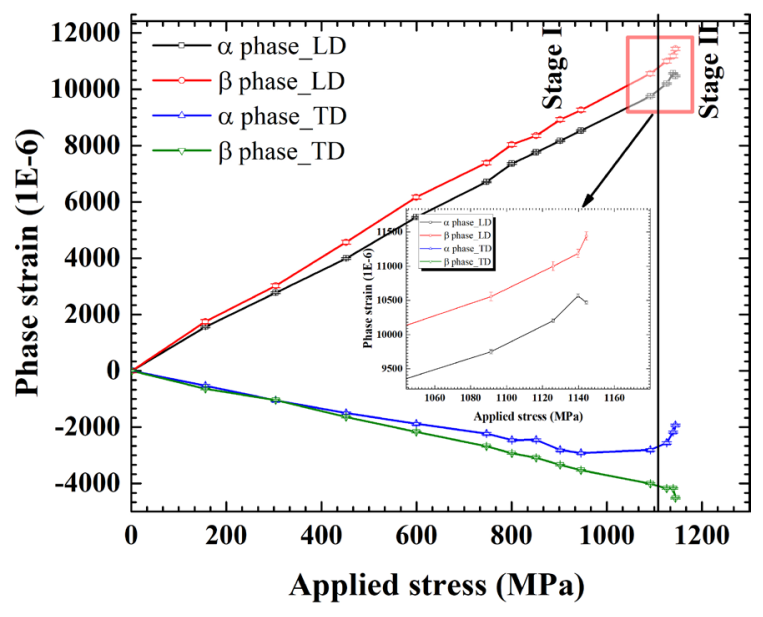

Fig. 4 The phase strain vs. applied stress at the LD and TD.

\subsection{Deformation microstructure}

Fig. 5a shows the EBSD characterized orientation mapping of deformed LM. Due to relatively small plastic strain, the shapes of $\beta$ phase grains have not changed a lot. The low angle grain boundaries (LAGBs) are observed in the $\beta$ matrix as indicated with black lines in Fig.5a. The formation of LAGBs could be ascribed to lattice rotation during deformation. In the case of $\alpha$ phase, the LAGBs are also observed within individual $\alpha$ lamellas. The kinking of $\alpha$ lamella appears when the length direction of $\alpha$ lamella is almost perpendicular to the LD. The local misorientation within individual $\alpha$ phase lamella shows that there is an orientation gradient in the $\alpha$ lamella as shown in Fig. $5 \mathrm{~b}$.

Fig. 5 EBSD analysis of deformed LM at a tensile strain of 0.05: (a) orientation mapping of constituent phases and (b) local misorientation distribution within $\alpha$ lamellas.

\section{Discussion}

According to the measured lattice strain, stress partitioning between $\alpha$ and $\beta$ phase could be clearly evaluated, which is helpful to understand the contribution of $\alpha$ and $\beta$ phase to mechanical properties of the Ti-55531 alloy with LM. The phase stress could be evaluated according to General Hooke's law, i.e.

$\sigma_{11}=E_{h k J} /\left(1+\nu_{h k j}\right)\left(e_{11}^{h k J}+\nu_{h k J} /\left(1-2 \nu_{h k j}\right)\left(\epsilon_{11}+\varepsilon_{22}+\varepsilon_{33}\right)\right)$

(3)

where $E_{h k J}, \nu_{h k J}, \varepsilon_{11}^{h k J}$ represents diffraction elastic constant, Poisson's ratio and lattice strain of considered $\{h k l\}$ reflection at the LD, respectively. The $\varepsilon_{22}^{\text {hkJ }}, \varepsilon_{33}^{\text {hkJ }}$ represents the lattice strain at the TD and ND (normal direction), respectively. Under the circumstance of plane stain, assume that $\varepsilon_{22}^{h k I}=\varepsilon_{33}^{h k}$. The lattice strain of $\{211\}_{\beta}$ and $\{1011\}$ is selected to calculate the phase stress of $\beta$ matrix and $\alpha$ precipitates as both selected reflections are insensitive to intergranular lattice strain. The stress partitioning between $\alpha$ and $\beta$ phase depends on the phase volumes and phase stress of each constituent phase according to mixture law, i.e.,

$\sigma_{\text {applied }}=f_{\alpha} \sigma_{\alpha}+f_{\beta} \sigma_{\beta}$

(4)

The stress partitioning between $\alpha$ and $\beta$ phase is illustrated in Fig. 6a. An agreement between calculated applied stress and experimental applied stress is observed. It is shown that $\beta$ matrix is subjected to higher stress than $\alpha$ precipitate during the plastic deformation. The stress partitioning between $\alpha$ and $\beta$ phase is dependent on elastic-plastic deformation behavior. In the present work, it is observed that the elastic properties of $\{1011\}$ and $\{211\}$ are nearly the same $\left.\left(E_{\{10 i 1}\right\}=114.6 \mathrm{GPa}, E_{\{211\}}=108.4 \mathrm{GPa}\right)$. In addition, the strain could cause the broadening of diffraction peaks, and the probable cause is the increase of dislocations. Fig. $6 \mathrm{~b}$ shows the evolution of full width at half maximum (FWHM) of diffraction peaks with the increase of applied stress. Upon a sharp increase at the onset of plasticity deformation, the FWHM of considered $\{\mathrm{hkl}\}$ reflections continue to increase during the plastic deformation. As the broadening of diffraction peaks likely caused by strain, it could be speculated that there is a discrepancy in dislocation density between $\alpha$ and $\beta$ phase. As a result, the $\beta$ phase is subjected to higher stress than $\alpha$ phase.

The intergranular and interphase microstress is generated with the increase of plastic strain. The detailed methods on the calculation of intergranular and interphase microstresses could be found in Ref. [7]. Fig. 6c plots the intergranular and interphase microstress as a function of applied stress. It is found that the intergranular microstress of $\beta$ phase is predominant among these microstresses. The intergranular stress causes a remarkable difference in the lattice strains between different oriented grains, which has been confirmed by previous research. S. Hemery et al. also found the highly heterogeneous elastic and plastic strain field in Ti-5553 alloy, and the root cause is identified as the elastic anisotropy of $\beta$ phase [3]. The intergranular microstress could lead to earlier crack initiation, and the intergranular crack mode has been confirmed by D.W. Becker et al [8]. The microstress characterization results by in-situ neutron diffraction are helpful for the understanding of fracture mechanism. 

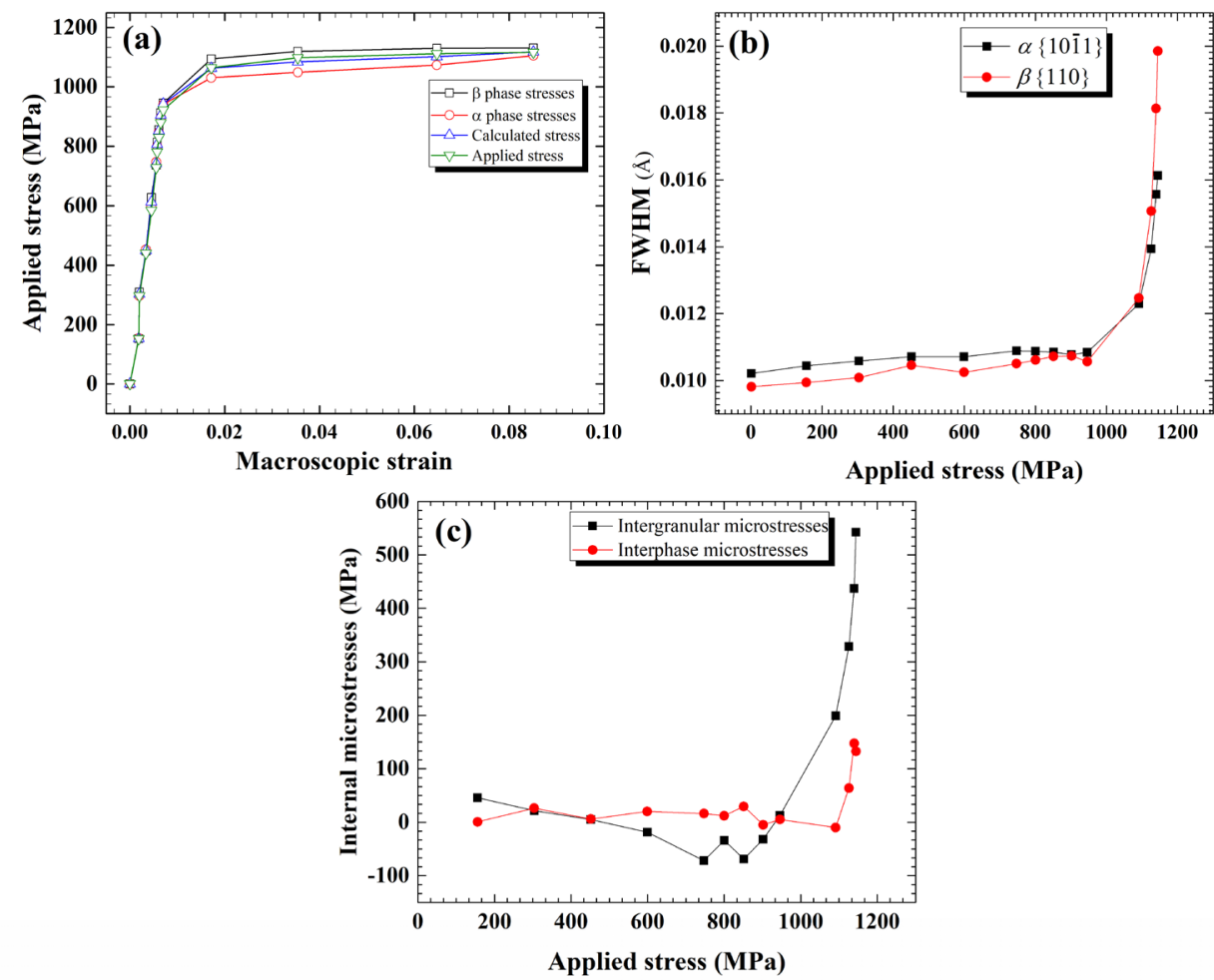

Fig. 6 (a) stress partitioning between $\alpha$ and $\beta$ phase, and (b) evolution of intergranular and interphase microstress.

\section{Conclusions}

In summary, the micromechanical behaviors of Ti-55531 alloy with lamellar microstructure were investigated by in-situ neutron diffraction. The stress partitioning between $\alpha$ precipitates and $\beta$ matrix was clearly illustrated. The $\beta$ matrix is subjected to higher phase stress than $\alpha$ precipitates. The intergranular and interphase microstress is generated during the deformation. Among these microstresses, the intergranular microstress is predominant. Combining the in-situ neutron diffraction characterization with microstructure characterization, the stress partitioning and anisotropy deformation behavior of oriented grains are quantitatively expressed. The particular attention to the contribution of $\beta$ phase may provide a valuable guide for further microstructure optimization of Ti-55531 alloy.

\section{Acknowledgements}

This work was supported by the Key Research and Development Program of China (Grants No. 2016YFB0701302). Neutron diffraction experiment was performed at BL19 in the Materials and Life Science Facility of J-PARC under experimental NO. 2018A0047.

\section{Reference}

[1] D. Banerjee, Acta Materialia, 61 (2013) 844-879.

[2] C. Li, J. Chen, W. Li, J. He, W. Qiu, Y. Ren, J. Chen, J. Chen, Journal of Alloys and Compounds, 627 (2015) 222-230.

[3] S. Hémery, P. Villechaise, Acta Materialia, 141 (2017) 285-293.

[4] N. Jia, Z.H. Cong, X. Sun, S. Cheng, Z.H. Nie, Y. Ren, P.K. Liaw, Y.D. Wang, Acta Materialia, 57 (2009) 3965-3977.

[5] Z. Ji, Y. Chen, Y. Qiang, C. Shen, H. Li, Materials Science and Engineering: A, 723 (2018) 29-37.

[6] R. Oishi, M. Yonemura, Y. Nishimaki, S. Torii, A. Hoshikawa, T. Ishigaki, T. Morishima, K. Mori, T. Kamiyama, Nuclear Instruments and Methods in Physics Research Section A: Accelerators, Spectrometers, Detectors and Associated Equipment, 600 (2009) 94-96.

[7] J. Johansson, M. Oden, X.-H. Zeng, Acta Materialia, 47 (1999) 2669-2684.

[8] D. Becker, W. Baeslack, Welding Journal, 59 (1980) 85-92. 